\title{
Private and Public Dimensions of Health Research Regulation
}

\author{
Introduction
}

\author{
Graeme Laurie
}

It is a common trope in discussions of human health research, particularly as to its appropriate regulation, to frame the analysis in terms of the private and public interests that are at stake. Too often, in our view, these interests are presented as being in tension with each other, sometimes irreconcilably so. In this section, the authors grapple with this (false) dichotomy, both by providing deeper insights into the nature and range of the interests in play, as well as by inviting us to rethink attendant regulatory responses and responsibilities. This is the common theme that unites the contributions.

The section opens with the chapter from Postan (Chapter 23) on the question of the return of individually relevant research findings to health research participants. Here an argument is made - adopting a narrative identity perspective - that greater attention should be paid to the informational interests of participants, beyond the possibility that findings might be of clinical utility. Set against the ever-changing nature of the researcher-participant relationship, Postan posits that there are good reasons to recognise these private identity interests, and, as a consequence, to reimagine the researcher as interpretative partner of research findings. At the same time, the implications of all of this for the wider research enterprise are recognised, not only in resource terms but also with respect to striking a defensible balance of responsibilities to participants while seeking to deliver the public value of research itself.

As to the concept of public interest per se, this has been tackled by Sorbie in Chapter 6, and various contributions in Section IB have addressed the role and importance of public engagement in the design and delivery of robust health research regulation. In this section, several authors build on these earlier chapters in multiple ways. For example, Taylor and Whitton (Chapter 24) directly challenge the putative tension between public and private interests, arguing that each is implicated in the other's protection. They offer a reconceptualisation of privacy through a public interest lens, raising important questions for existing laws of confidentiality and data protection. Their perspective requires us to recognise the common interest at stake. Most uniquely, however, they extend their analysis to show how group privacy interests currently receive short shrift in health research regulation, and they suggest that this dangerous oversight must be addressed adequately because the failure to recognise group privacy interests might ultimately jeopardise the common public interest in health research.

Starkly, Burgess (Chapter 25) uses just such an example of threats to group privacy - the care. data debacle - to mount a case for mobilising public expertise in the design of health research regulation. Drawing on the notion of deliberative public engagement, he demonstrates how this process cannot only counter asymmetries of power in the structural design of regulation but also 
how the resulting advice about what is in the public interest can bring both legitimacy and trustworthiness to resultant models of governance. This is of crucial importance, because as he states: '[i]t is inadequate to assert or assume that research and its existing and emerging regulation is in the public interest'. His contribution allows us to challenge any such assertion and to move beyond it responsibly.

The last two contributions to this section continue this theme of structural reimagining of regulatory architectures, set against the interests and values in play. Vayena and Blassime (Chapter 26) offer the example of Big Data to propose a model of adaptive governance that can adequately accommodate and respond to the diverse and dynamic interests. Following principlesbased regulation as previously discussed by Sethi in Chapter 17, they outline a model involving six principles and propose key factors for their implementation and operationalisation into effective governance structures and processes. This form of adaptive governance mirrors the discussions by Kaye and Prictor in Chapter 10. Importantly, the factors identified by the current authors - of social learning, complementarity and visibility - not only lend themselves to full and transparent engagement with the range of public and private interests, they require it. In the final chapter of this section, Brownsword (Chapter 27) invites us to address an overarching question that is pertinent to this entire volume: how are the interests in pushing forward with research into potentially beneficial health technologies to be reconciled with the heterogeneous interests of the concerned who seek to push back against them?' His contribution is to push back against the common regulatory response when discussing public and private interests: namely, to seek a 'balance'. While not necessarily rejecting the balancing exercise as a helpful regulatory device at an appropriate point in the trajectory of regulatory responses to a novel technology, he implores us to place this in 'a bigger picture of lexically ordered regulatory responsibilities'. For him, morally and logically prior questions are those that ask whether any new development - such as automated healthcare - poses threats to human existence and agency. Only thereafter ought we to consider a role for the balancing exercise that is currently so prevalent in human health research regulation.

Collectively, these contributions significantly challenge the public/private trope in health research regulation, but they leave it largely intact as a framing device for engaging with the constantly changing nature of the research endeavour. This is helpful in ensuring that on-going conversations are not unduly disrupted in unproductive ways. By the same token, individually these chapters provide a plethora of reasons to rethink the nature of how we frame public and private interests, and this in turn allows us to carve out new pathways in the future regulatory landscape. Thus:

- Private interests have been expanded as to content (Postan) and extended as to their reach (Taylor and Whitton).

- Moreover, the implications of recognising these reimagined private interests have been addressed, and not necessarily in ways resulting in inevitable tension with appeals to public interest.

- The content of public interest has been aligned with deliberative engagement in ways that can increase the robustness of health research regulation as a participative exercise (Burgess).

- Systemic oversight that is adaptive to the myriad of evolving interests has been offered as proof of principle (Vayena and Blassime).

- The default of seeking balance between public and private interests has been rightly questioned, at least as to its rightful place in the stack of ethical considerations that contribute to responsible research regulation (Brownsword). 